\title{
Theoretical introspection
}

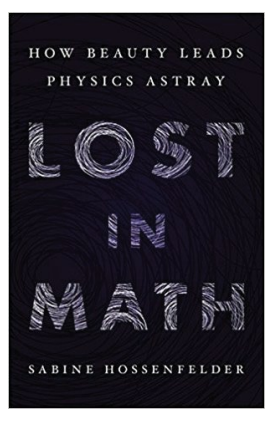

Lost in Math: How Beauty Leads Physics Astray

by Sabine Hossenfelder

BASIC BOOKS: 2018 304 PP. US\$30

$\mathrm{T}$ he foundations of physics leave little room for decoration. All that counts is the scaffold: space, time, 25 elementary particles - and the laws that govern their interactions. Propped up by mathematical formalism, this is a framework built on past successes: predicted particles that were later observed in experiments, and theories encompassing phenomena so challenging to detect that empirical evidence seemed obstinately elusive - as in the case of gravitational waves. Of course there are open questions in theoretical physics, but that's what makes research worth pursuing. And what could go wrong on the path to further progress given the field's glorious past?

More than a few things, says theoretical physicist Sabine Hossenfelder. That the present scaffold is shaky has been known for a while: improving it is a subject of active research. But Hossenfelder worries that hers will be the generation that fails to advance our fundamental understanding of physical reality. In her first popular science book, she invites the reader on a journey through theory development in physics: how it works, why she thinks it's broken despite and perhaps because of - its former glory, and what might be done to rescue it.

Hossenfelder argues that the fruitful collaboration between theory and experiment in high-energy physics might have hit a roadblock: it's been 45 years since the last theoretical prediction of a new fundamental particle that was later confirmed by experiment. The top quark, postulated in 1973 , was subsequently observed in 1995; experimental signatures of the Higgs boson, predicted in the early 60 s, were only found in 2012. The truth is, empirical observations are increasingly difficult to gather. The data required to inspire or refine theories call for adequate financing, as well as skilled fleets responsible for complex instrumentation and ever-increasing dataset sizes.
What does it mean for a theory to be elegant? Why would theorists care about a model's beauty or lack thereof? These are difficult questions even for a practicing theoretical physicist, which is why Hossenfelder returns to them throughout the book. She doesn't provide definitive answers, but she quotes scientists past and present who praise the beautiful symmetries that lead to unifying frameworks and despise unnatural theories with cherrypicked assumptions. Aesthetic criteria shouldn't be dangerous per se; yet none of the evidence gathered in the book proves such principles infallible. The author's concern is that her colleagues might have slipped into a blind reliance on beauty as a guide for theory development, turning it into the mathematical criterion that it was never meant to be.

Hossenfelder's depiction of the current state of affairs in theoretical particle physics might look dire, but the book isn't a defeatist's lament. To me it came across as a rather honest presentation of a scientific community, of its strengths and contradictions. And Hossenfelder is a savvy guide: she introduces concepts and terminology when needed, taking a pragmatic approach that allowed me to appreciate the technicalities in a meaningful context. I learned about the criteria that guide model-building theorists, and became aware of the confusion some of them experience when data in support of their predictions - think supersymmetric partners - fail to show up.

The structure of the book is both refreshing and engaging: fact-based sections are intertwined with Hossenfelder's musings and conversations with fellow physicists. I think this choice reflects the author's complex feelings about research in theoretical physics, because Hossenfelder is not an external observer: she is a scientist struggling to make sense of the course taken by her chosen field. Her criticisms of the current practices among fellow model builders become sharper - extending well beyond her research field by the end of the book - as she ponders, exchanges views and draws conclusions, as much to the reader's benefit as her own.

Here one might be tempted to identify theorists' obsession for beautiful models as the biggest problem plaguing the field (at least according to Hossenfelder) - and one would then be mistaken. As it turns out, physicists are human: they like being part of a group and telling each other they're right, and they can be highly effective at filtering out dissonant voices. The presence of such cognitive biases becomes more obvious when Hossenfelder talks to researchers working outside of the mainstream, such as Garrett Lisi and Xiao-Gang Wen. Lisi is a surfercum-physicist who developed a 'simple theory of everything' based on the E8 Lie group from his retreat in Maui, while Wen advocates a unifying framework in which quantum bits substitute elementary particles. When asked about the reactions to their ideas among other physicists, they provide similar feedback: interest is tepid, if it exists at all, and most alternative approaches are ignored at best.

I appreciated how Hossenfelder included conversations with veterans such as Steven Weinberg and Frank Wilczek alongside interviews with the likes of Lisi and Wen. This choice reveals the plurality of voices participating in the same quest for knowledge. Still, it seems that sharing a goal isn't all that's needed to build a healthy scientific community. In theoretical physics, young and ambitious researchers appear to be encouraged to pick a school of thought with some prior backing from the community - unusual ideas are risky. But are they really?

I suspect Hossenfelder's analysis will be as easy to support as it will be likely to spark disagreement. Whereas some might feel that she makes fun of her own lot (when she provides a step-by-step 'recipe' for making up new particles, for example), I never found her tone unnecessarily caustic or her critique gratuitous. She seems chiefly moved by her respect for science and knowledge, and she wishes to provoke a reaction - or rather a back-reaction - to her thoughts. Hossenfelder is hopeful about the future of physics; her positive tone in closing the book strengthens this impression. After all, wandering theorists may not (yet) be lost.

Reviewed by Gaia Donati Associate Editor at Nature

Published online: 4 July 2018 https://doi.org/10.1038/s41567-018-0198-5 